Delft University of Technology Software Engineering Research Group Technical Report Series

\title{
Using Association Rules to Study the Co-evolution of Production \& Test Code
}

Zeeger Lubsen, Andy Zaidman, Martin Pinzger

Report TUD-SERG-2009-015

T̛UDelft 
TUD-SERG-2009-015

Published, produced and distributed by:

Software Engineering Research Group

Department of Software Technology

Faculty of Electrical Engineering, Mathematics and Computer Science

Delft University of Technology

Mekelweg 4

2628 CD Delft

The Netherlands

ISSN 1872-5392

Software Engineering Research Group Technical Reports:

http://www.se.ewi.tudelft.nl/techreports/

For more information about the Software Engineering Research Group:

http://www.se.ewi.tudelft.nl/

Note: Paper accepted for publication in the proceedings of the 6th International Working Conference on Mining Software Repositories (MSR 2009)

(C) copyright 2009, by the authors of this report. Software Engineering Research Group, Department of Software Technology, Faculty of Electrical Engineering, Mathematics and Computer Science, Delft University of Technology. All rights reserved. No part of this series may be reproduced in any form or by any means without prior written permission of the authors. 


\section{Using Association Rules to Study the Co-evolution of Production \& Test Code}

\author{
Zeeger Lubsen \\ Software Improvement Group \\ The Netherlands \\ z.lubsen@sig.nl
}

\author{
Andy Zaidman, Martin Pinzger \\ Delft University of Technology \\ The Netherlands \\ \{a.e.zaidman, m.pinzger\}@tudelft.nl
}

\begin{abstract}
Unit tests are generally acknowledged as an important aid to produce high quality code, as they provide quick feedback to developers on the correctness of their code. In order to achieve high quality, well-maintained tests are needed. Ideally, tests co-evolve with the production code to test changes as soon as possible. In this paper, we explore an approach based on association rule mining to determine whether production and test code co-evolve synchronously. Through two case studies, one with an open source and another one with an industrial software system, we show that our association rule mining approach allows one to assess the co-evolution of product and test code in a software project and, moreover, to uncover the distribution of programmer effort over pure coding, pure testing, or a more test-driven-like practice.
\end{abstract}

\section{Introduction}

The development of high quality software systems is a complex process; maintaining an existing system is often no less challenging. Runeson notes that automated unit testing ${ }^{1}$ can be an effective countermeasure for difficulties encountered during software maintenance [5]. Also TestDriven Development (TDD) and test-driven refactoring [4] can play an important role here.

The quality of the tests - and by consequence the added value for maintenance activities - greatly depends on the effort that the developers put into writing and maintaining tests. Traditional test suite quality measures are typically not good at indicating the long term quality or "test health" of a test suite [7]. As such, we have no insight into (1) how well test code was adapted to previous changes in the production code, (2) the current structure of the test code,

\footnotetext{
${ }^{\star}$ This work is described in more detail in the MSc thesis of Zeeger Lubsen [3].

${ }^{1}$ xUnit Testing Frameworks: http://www.xunit.org
}

and (3) how easy it will be to perform maintenance on both the production and the test code in the future.

This missing insight has motivated us to investigate the co-evolution of production and test code. In our previous work, we introduced the Change History View [7] to observe and perform a qualitative analysis of the co-evolution of production and test code by mining version control data. While change history views provide sufficient insights into the co-evolution of production of test code they require a fair amount of human effort to understand and interpret.

In this paper, we address this shortcoming by adding a quantitative analysis approach to study the co-evolution of production and test code. In particular, we investigate whether association rule mining can be applied to study the co-evolution of test and production code and provide answers to the following research questions:

RQ1: Can association rule mining be used to find evidence of co-evolution of production and test code?

RQ2: Following RQ1, can we find measures to assess the extent to which product and test code co-evolves?

RQ3: Can different patterns of co-evolution be observed in distinct settings, for example, open source versus industrial software systems?

We address these research questions by means of two case studies. The first case study is on Checkstyle, an open source system that checks whether code adheres to a coding standard. The second case study is on an industrial software system from the Software Improvement Group (SIG) ${ }^{2}$.

\section{Production and test class co-evolution}

Within the realm of data mining, we have chosen to use association rule mining, because this technique allows us to identify instances of logical coupling between classes [8], in particular between production and test classes. For this paper, production code/classes refer to Java classes and test code/classes to jUnit test classes.

\footnotetext{
${ }^{2}$ Software Improvement Group, Amsterdam, The Netherlands. http://www.sig.nl
} 
The basic idea of our approach is to use association rule mining to study the co-evolution of test and production code. The change history of test and production classes, in particular commit transactions, form the input to our approach. Information about commit transactions is obtained from versioning repositories, such as, the concurrent versions systems (CVS) or Subversion (SVN).

\subsection{Association rule mining}

Formally, an association rule is a statistical description of the co-occurrence of elements in the change history that constitute the rule in the change history [1]:

Definition 1 Given a set of items $I=I_{1}, I_{2}, \ldots, I_{m}$ and a database of transactions $D=t_{1}, t_{2}, \ldots, t_{n}$ where $t_{i}=$ $I_{i 1}, I_{i 2}, \ldots, I_{i k}$ and $I_{j k} \in I$, an association rule is an implication of the form $A \Rightarrow B$ where $A, B \subset I$ are sets of items called itemsets and $A \cap B=\emptyset$.

The left-hand side of the implication is called the antecedent, and the right-hand side is called the consequent of the rule. An association rule expresses that the occurrence of $A$ in a transaction statistically implies the presence of $B$ in the same transaction with some probability.

In our approach, we consider association rules that express a binary relation between classes, as we are looking for relations between individual production classes (PC) and test classes (TC). For example, consider the SVN transaction $\left\{T C_{1}, P C_{1}, P C_{2}\right\}$ committing changes to the test class $T C_{1}$, and the two production classes $P C_{1}$ and $P C_{2}$. Computing all pairs we get the following binary association rules: $\left\{T C_{1} \rightarrow P C_{1}\right\}, \quad\left\{P C_{1} \rightarrow T C_{1}\right\}, \quad\left\{P C_{2} \rightarrow T C_{1}\right\}$, $\left\{T C_{1} \rightarrow P C_{2}\right\}, \quad\left\{P C_{1} \rightarrow P C_{2}\right\}, \quad\left\{P C_{2} \rightarrow P C_{1}\right\}$,

For a transaction involving $n$ classes we obtain $n *(n-1)$ binary association rules. We take into account inverse association rules, because the inverse rules can have a different probability, as we explain below.

\subsection{Co-evolution rules}

In order to analyze the testing practices for an entire system, we need a high-level overview of the development and testing activities of the software system. For that, we classify binary association rules according to rules that deal (1) solely with production code, (2) solely with test code, and (3) that deal with both production and test code. Table 1 shows this classification in detail.

While PT comprises association rules between product and test code the sub-classes refine this set by taking the direction of rules into account. The direction of rules comes into play when calculating the interestingness of an association rule. Furthermore, we introduce two categories

\begin{tabular}{|c|c|}
\hline Class & Association rule \\
\hline TOTAL & The collection of all found association rules. \\
\hline PROD & $\{$ ProductionClass $\Rightarrow$ ProductionClass $\}$ \\
\hline & Rules that only associate production classes. \\
\hline TEST & $\{$ TestClass $\Rightarrow$ TestClass $\}$ \\
\hline & Rules that only associate test classes. \\
\hline PT & $\begin{array}{l}\text { Rules that associate production-test pairs, which we can } \\
\text { subdivide into: }\end{array}$ \\
\hline $\mathrm{P} 2 \mathrm{~T}$ & $\begin{array}{l}\{\text { TestClass } \Rightarrow \text { ProductionClass }\} \text {. These rules express } \\
\text { that a change in production class implies a change in } \\
\text { test class with some probability. }\end{array}$ \\
\hline $\mathrm{T} 2 \mathrm{P}$ & $\begin{array}{l}\{\text { ProductionClass } \Rightarrow \text { TestClass }\} \text {. These rules express } \\
\text { that a change in test class implies a change in produc- } \\
\text { tion class with some probability. }\end{array}$ \\
\hline MP2T & $\begin{array}{l}\text { Matching production to test rules; } \mathrm{P} 2 \mathrm{~T} \text { rules where the } \\
\text { antecedent and the consequent can be matched to be- } \\
\text { long together as unit test and class-under-test. These } \\
\text { rules express that a change in production code implies } \\
\text { a change in test code with some probability. }\end{array}$ \\
\hline MT2P & The counterpart of MP2T. \\
\hline
\end{tabular}

Table 1. Classification of association rules.

containing rules that denote commit transactions in which a test class has been matched to a production class. For each commit transaction these rules are obtained by comparing the file names of product and test classes. For the comparison we rely on naming convention for test classes and use straightforward string matching. For example, a production class Class.java is matched with the test class ClassTest.java.

\subsection{Co-evolution metrics}

Typically, association rule mining is used to search for rules that are "interesting" or "surprising". In our case, we seek to find a global view on the entire change history of source files (i.e., top-level Java classes) of a software project. As such, we are mainly interested in the total number of rules that associate production and test classes and how "interesting", i.e., how strong the statistical certainty of these rules is. In the following we explore a number of standard rule significance and interest measurements to measure co-evolution between production and test classes in a software system.

The metrics presented in Table 2 allow us to reason about the significance and interest of single association rules. To get an overall understanding of how production and test code co-evolves in a software system we use straight forward descriptive statistics with boxplots. Boxplots provide a five-number summary of the distribution of significance and interest metric values. The sample minimum and maximum define the range of the values, while the median designates the central tendency of the distribution. The lower and upper quartile allow reasoning about the standard deviation and together with the median about the skewness of metric values. 


\begin{tabular}{|c|c|c|c|}
\hline Metric & Probability & Implementation & Interpretation \\
\hline $\operatorname{support}(A \Rightarrow B)$ & $P(A, B) n$ & count & $\begin{array}{l}\text { The fraction of commits in which the itemset }\{A, B\} \text { appears in the change } \\
\text { history. Abbreviated as: } \mathrm{s}(A \Rightarrow B) \text {. }\end{array}$ \\
\hline $\operatorname{confidence}(A \Rightarrow B)$ & $P(B \mid A)$ & $\frac{s(A, B)}{s(A)}$ & $\begin{array}{l}\text { The ratio of the number of transactions that contain classes }\{A \cup B\} \text { to the } \\
\text { number of transactions that contain class } A \text {. This measure is not symmetrical. }\end{array}$ \\
\hline $\operatorname{interest}(A \Rightarrow B)$ & $\frac{P(A, B)}{P(A) P(B)}$ & $\frac{s(A, B) n}{s(A) s(B)}$ & $\begin{array}{l}\text { Measures the correlation between the two classes } A \text { and } B \text {, i.e., how many times } \\
\text { more often class } A \text { and } B \text { are contained in a commit transaction then expected } \\
\text { if they were statistically independent. This measure is symmetrical. }\end{array}$ \\
\hline $\operatorname{conviction}(A \Rightarrow B)$ & $\frac{P(A) P(\neg B)}{P(A, \neg B)}$ & $\frac{s(A) n-\frac{s(A) s(B)}{n}}{s(A)-s(A, B)}$ & $\begin{array}{l}\text { Is a measure of the implication that whenever class A is committed class B is } \\
\text { also committed. This measure is not symmetrical. }\end{array}$ \\
\hline
\end{tabular}

Table 2. Metrics for individual association rules.

These metric-values help us in interpreting the interestingness of the association rule classes that we have defined in Section 2.2. If a rule appears in almost all commits, its support is close to $100 \%$. While this is unlikely to happen for all commits, finding outliers that exhibit a support close to $100 \%$ is interesting, e.g., as they indicate a possible bad design choice if two classes have been changed together that often. The confidence-metric is tightly related to the concept of co-evolution. It represents the certainty with which one can expect, for example, when the product class is changed that also the test class is changed. Confidence values higher than 0.5 give a clear indication of co-evolution between classes. The interest becomes higher when the rule frequently holds. As for conviction, highquality rules (those that hold $100 \%$ of the time) have a value of $\infty$, while the less interesting rules have a value that approaches 1 (rules from completely unrelated items have a metric-value of 1) [2].

Co-evolution of production and test classes is indicated by rules in PT and its subclasses with significant support, high confidence, interest, and conviction. Separate evolution of product and test classes is indicated by rules in PROD and TEST with significant support, high confidence, interest, and conviction. If the majority of PROD, TEST, and PT rules has low support, we conclude that there is no structural co-evolution between classes.

In addition to the association rule interest measures, we introduce several metrics to measure the extent to which product classes are covered by test classes. The set of metrics is described in Table 3.

These coverage metrics allow us to get an insight into the testing strategy. More precisely, a high ratio of PCC and TCC indicates that many production class and test class pairs are changed together. On the other hand, high ratios of mPCC and mTCC indicate that the co-change is structural.

\section{Preliminary results}

We tried out our approach using two software systems: Checkstyle, an open source coding standard checker, and an industrial software system provided to us by the Software

\begin{tabular}{|c|c|}
\hline Metric & Description \\
\hline PCC & $\begin{array}{l}\text { Production class coverage. The average number } \\
\text { of test classes that are changed per changed pro- } \\
\text { duction code class. This number is calculated by } \\
\qquad|P 2 T|\end{array}$ \\
\hline MPCC & $\begin{array}{l}\text { \#productionclasses. } \\
\text { Matching production class coverage. The percent- } \\
\text { age of production classes that co-evolve with their } \\
\text { matched unit test class. This number is calculated by } \\
\frac{|m P 2 T|}{\# \text { productionclasses }} \text {. }\end{array}$ \\
\hline $\mathrm{TCC}$ & $\begin{array}{l}\text { Test class coverage. The average number of produc- } \\
\text { tion code classes that are changed per changed unit test } \\
\text { class. This number is calculated by } \frac{|T 2 P|}{\#+e s t c l a s s e s} \text {. }\end{array}$ \\
\hline MTCC & $\begin{array}{l}\text { Matching test class coverage. The percentage of } \\
\text { test classes that co-evolve with its matched produc- } \\
\text { tion class-under-test. This number is calculated by } \\
\frac{\mid \text { mT } 2 P \mid}{\# \text { testclasses. }}\end{array}$ \\
\hline
\end{tabular}

Table 3. Product-test class coverage metrics.

Improvement Group (SIG).

Checkstyle. For Checkstyle we saw that actual software development and testing are mainly two separate activities, which is mainly evidenced through the rule ratios. However, a possible complication that we came across when interpreting the results was the fact that there are some large commits of (mainly) production code, which dominate the rule ratios to a large extent, thereby perturbing the interpretation. These very large commits originate from automated code beautification operations (using Checkstyle).

During our interpretation, we also observed large differences between $\mathrm{mT} 2 \mathrm{P}$ and $\mathrm{mP} 2 \mathrm{~T}$ rules when studying the confidence and conviction rules. In particular, we saw that the statistical evidence for $\mathrm{mT} 2 \mathrm{P}$ rules was stronger than for $\mathrm{mP} 2 \mathrm{~T}$ rules. Closer inspection revealed this to be due to commits containing a larger number of production code classes than test code classes, thereby influencing the probabilities behind confidence and conviction.

Considering the average number of production and test classes that are changed together, we can say that in general not many production and test classes are co-evolved as evidenced by the very low PCC and TCC values. This is 
further underlined by the low $\mathrm{mPCC}$ and $\mathrm{mTCC}$ values.

SIG software system. In our industrial case study we observed that the SIG developers are following a development and testing strategy that resembles that of a test-driven development strategy. The first indication is given by the fact that the rule class ratios are fairly evenly distributed over PROD, TEST and PT.

Another important indicator for test-driven development are the rule coverage ratios for the SIG software system. Here we saw that for each production class that has been changed, also a significant number of test classes has been changed (and vice versa). This phenomenon is also structural, as also matched production and test class pairs have been changed together.

\section{Conclusion and future work}

In this paper we have made the following contributions:

- An approach using association rule mining to study the co-evolution of production and test code in a system using transactions obtained from version control.

- A set of co-evolution metrics including standard interest and strength association rule mining metrics to assess the extent to which product and test classes evolve.

- An evaluation with two case studies, one performed with the open source software project Checkstyle, and another one performed with an industrial software system provided by the Software Improvement Group. In both case studies, the findings have been evaluated and validated with the findings of our previous research and the original developers/maintainers of the software systems under study.

The two case studies that we performed have shown a greatly differing testing approach. In the case of Checkstyle, we saw a very mixed picture at first, since we observed that most of the commits are dominated by changes to production code. This is (1) due to the development style, where testing is mainly done in phases outside of regular development (this is true during the early development of Checkstyle), but also (2) due to a small number of large commits of production code that perturbs the rule classification (these large commits are due to code beautification). Our industrial case study, on the other hand, has shown a test-driven development approach to testing, evidenced by a large number of commits that contained both additions/changes to production and test code.

The analysis techniques that we have explored in this work prove to be useful for (retrospective) assessment of the unit test suite. A weak point of our approach, however, is the fact that changes to the testing practices over small periods of time will not yield noticeable differences in the results, as our technique summarizes the entire history.

Future work. We have identified a number of ideas to build upon this research.

- The use of an inter-transactional association rule mining algorithm, which allows to widen our analysis from a single commit to a window of commits that were made in a short amount of time [6].

- Traversing the change history with a sliding window, so that time-intervals can be studied more in depth, details become more clear and trends can be identified.

Acknowledgments. We would like to thank the Software Improvement Group for their support during this research and Bas Cornelissen and Bart Van Rompaey for proofreading this paper. Funding for this research came from the NWO Jacquard Reconstructor project and from the Centre for Dependable ICT Systems (CeDICT).

\section{References}

[1] R. Agrawal, T. Imielinski, and A. N. Swami. Mining association rules between sets of items in large databases. In Proceedings of the ACM SIGMOD International Conference on Management of Data, pages 207-216. ACM, 1993.

[2] S. Brin, R. Motwani, J. D. Ullman, and S. Tsur. Dynamic itemset counting and implication rules for market basket data. In Proc. of the International Conference on Management of Data (SIGMOD), pages 255-264. ACM, 1997.

[3] Z. Lubsen. Studying co-evolution of production and test code using association rule mining. Master's thesis, Software Engineering Research Group, Delft University of Technology, 2008.

[4] L. Moonen, A. van Deursen, A. Zaidman, and M. Bruntink. Software Evolution, chapter The interplay between software testing and software evolution, pages 173-202. Springer, 2008.

[5] P. Runeson. A survey of unit testing practices. IEEE Software, 23(4):22-29, 2006.

[6] A. K. H. Tung, H. Lu, J. Han, and L. Feng. Breaking the barrier of transactions: Mining inter-transaction association rules. In Proc. of the Int'l Conference on Knowledge Discovery and Data Mining (KDD), pages 297-300. ACM, 1999.

[7] A. Zaidman, B. van Rompaey, S. Demeyer, and A. van Deursen. Mining software repositories to study co-evolution of production and test code. In Proc. Int'l Conf. on Software Testing, Verification and Validation (ICST), pages 220-229. IEEE, 2008.

[8] T. Zimmermann, P. Weißgerber, S. Diehl, and A. Zeller. Mining version histories to guide software changes. In Int'l Conf. on Software Engineering (ICSE), pages 563-572. IEEE, 2004. 

TUD-SERG-2009-015 ISSN 1872-5392 